\title{
Gene expression patterns of chicken neuregulin 3 in association with copy number variation and frameshift deletion
}

\author{
Hideaki Abe $^{1 *}$ D, Daiki Aoya ${ }^{2}$, Hiro-aki Takeuchi ${ }^{3}$ and Miho Inoue-Murayama ${ }^{1,4}$
}

\begin{abstract}
Background: Neuregulin 3 (NRG3) plays a key role in central nervous system development and is a strong candidate for human mental disorders. Thus, genetic variation in NRG3 may have some impact on a variety of phenotypes in non-mammalian vertebrates. Recently, genome-wide screening for short insertions and deletions in chicken (Gallus gallus) genomes has provided useful information about structural variation in functionally important genes. NRG3 is one such gene that has a putative frameshift deletion in exon 2, resulting in premature termination of translation. Our aims were to characterize the structure of chicken NRG3 and to compare expression patterns between NRG3 isoforms.

Results: Depending on the presence or absence of the 2-bp deletion in chicken NRG3, 3 breeds (red junglefowl [RJF], Boris Brown $[\mathrm{BB}]$, and Hinai-jidori $[\mathrm{HJ}]$ ) were genotyped using flanking primers. In the commercial breeds (BB and $\mathrm{HJ}$ ), approximately $45 \%$ of individuals had at least one exon 2 allele with the 2-bp deletion, whereas there was no deletion allele in RJF. The lack of a homozygous mutant indicated the existence of duplicated NRG3 segments in the chicken genome. Indeed, highly conserved elements consisting of exon 1, intron 1, exon 2, and part of intron 2 were found in the reference RJF genome, and quantitative PCR detected copy number variation (CNV) between breeds as well as between individuals. The copy number of conserved elements was significantly higher in chicks harboring the 2-bp deletion in exon 2. We identified 7 novel transcript variants using total mRNA isolated from the amygdala. Novel isoforms were found to lack the exon 2 cassette, which probably harbored the premature termination codon. The relative transcription levels of the newly identified isoforms were almost the same between chick groups with and without the 2-bp deletion, while chicks with the deletion showed significant suppression of the expression of previously reported isoforms.

Conclusions: A putative frameshift deletion and CNV in chicken NRG3 are structural mutations that occurred before the establishment of commercial chicken lines. Our results further suggest that the putative frameshift deletion in exon 2 may potentially affect the expression level of particular isoforms of chicken NRG3.
\end{abstract}

Keywords: Alternative splicing, Copy number variation (CNV), Frameshift, Gallus gallus, Indel, Isoform, Neuregulin 3 (NRG3), Premature stop codon, Retained intron, RT-qPCR

\section{Background}

Recently, next generation sequencing has been used to identify insertion and deletion (indel) variation in chicken genomes [1]. The identification of short indels among 12 diverse chicken breeds has provided valuable information on genetic variation in genic, intergenic, and intronic regions. In terms of the identified coding indels, 1022 were predicted to cause frameshift mutations by non-

\footnotetext{
* Correspondence: habe2031@gmail.com

${ }^{1}$ Wildlife Research Center, Kyoto University, 2-24 Tanaka-Sekiden-cho, Sakyo,

Kyoto 606-8203, Japan

Full list of author information is available at the end of the article
}

triplet indels, leading to the generation of premature termination codons (PTCs). Indels, especially frameshift indels, are of great importance for their potential to alter gene function by the creation of alternative splicing events [2]. Our pilot survey showed that chicken neuregulin 3 (NRG3) is one of these important genes that contain a putative frameshift mutation in a coding exon (Chr6; 3,200,391 in ENSGALG00000002327).

NRG3 is a neuronal-enriched growth factor that binds specifically to the ErbB4 receptor tyrosine kinase in the developing mammalian forebrain [3]. NRG3 plays multiple roles in the development of the embryotic central 
nervous system by regulating the migration and patterning of neural progenitor cells [4]. Similar to neuregulin 1 (NRG1), NRG3 has been identified as a strong candidate molecule for neurodevelopment disorders accompanied with cognitive and behavioral abnormalities [5-7].

Many studies have been carried out to determine the transcript variants of human NRG3 cloned from various brain regions. NRG3 undergoes extensive alternative splicing and utilization of its first and second exons [8,9]. Two single nucleotide polymorphisms located in the $5^{\prime}$ region of NRG3 were shown to have a critical effect on the selection of alternative first exons [10]. These findings on alternative exon selection, together with those derived from NRG1 [11, 12], suggest that there may be alternative transcription start sites in the upstream region of chicken NRG3.

Our preliminary survey indicated the existence of a partial duplication $(4.4 \mathrm{~kb})$ of chicken NRG3 in the reference genome assembly (galGal4), suggesting that copy number variation $(\mathrm{CNV})$ may be another source of structural variation in this gene. CNV is the most prevalent type of structural variation that generally harbors relatively long duplications or deletions ( $\geq 1 \mathrm{~kb})$ [13]. In the mouse genome, $5.5 \%$ of detected $\mathrm{CNV}$ overlapped with some part of a gene [14], thereby occasionally changing gene structure and transcription patterns. Accumulating evidence suggests that CNV loci harboring duplications or deletions have affected gene expression due to dosage compensation [15] or dosage sensitivity [16]. Even a partial gene duplication or deletion may have a pivotal impact on gene expression, especially when a CNV locus encompasses important sequence elements for transcription such as alternative transcription start sites and cis-regulatory factors [17].

Here, we present data from a comprehensive analysis of chicken NRG3, focusing on the association between structural variation and gene expression patterns. It is of great interest to examine whether a duplication that occurred in the ancestor of the chicken (i.e., red junglefowl $[R J F]$ ) would increase or decrease its copy number in commercial breeds under selective pressure, and whether increases and decreases in copy number would coincide with the up- or down-regulation of known NRG3 isoforms.

\section{Results}

\section{Structure of chicken NRG3}

The structure of chicken NRG3 (chr6: 2,926,344 - 3,201,956; ENSGALG00000002327) was investigated by BLAT search [18] against the RJF genome assembly (galGal4). The upstream sequence of chicken NRG3 contained several highly conserved elements in the $5^{\prime}$ untranslated region (UTR), coding region of exon 1 , and $5^{\prime}$ region of intron 1 (Fig. 1). Alignment of these elements with orthologous sequences showed preservation of the reading frame in the coding region (Additional file 1: Figure S1). Besides these elements, a 5' truncated exon ("Exon1_long" embedded in the CLASS II isoforms) was found between E1c and E1d. The exact locations and sequence similarities of exon 1 and intron 1 are described in Table 1. In addition, a longer stretch of a duplicated element containing a highly conserved exon 1, intron 1, exon 2, and partial intron 2 (4.4 kb in total; hereafter, $\Psi$ NRG3) was found in an unknown location of the RJF reference genome (JH375293).

\section{Different frequencies of the 2-bp deletion allele among chicken breeds}

Polymerase chain reaction (PCR) amplification using flanking primers (NRG3_longF and NRG3_commonR) generated a band with the expected size in all samples tested (Fig. 2a), while when an internal reverse primer (NRG3_dupR2) overlapping with the 2-bp deletion was used for amplification, faint bands appeared in several Boris Brown (BB) and Hinai-jidori (HJ) samples (Fig. 2b). PCR amplification with a fluorescently-labeled forward primer (NRG_intF) and Sanger sequencing confirmed that these faint bands had the 2-bp deletion in exon 2, as reported previously [1]. This PCR yielded 2 different patterns in peak detection: a single strong peak was detected in wild-type (hereafter, WT; Fig. 2c), otherwise, a minor 2-bp shorter peak appeared with a much higher $W T$ peak (hereafter, $\boldsymbol{d e l}$; Fig. 2d). The frequency of the $\boldsymbol{d e l}$ allele was largely different between commercial (BB and $\mathrm{HJ}$ ) and primitive chickens (i.e., RJF). BB and HJ showed the same level of $\boldsymbol{d e l}$ frequency, whereas the $\boldsymbol{d e l}$ allele was not detected in RJF (Table 2).

\section{Abundance of transcription factor binding sites in the conserved region of intron 1}

LASAGNA-Search $2.0[19,20]$ identified a total of 1655 transcription factor binding sites (TFBSs) in intron 1 of chicken NRG3. The distribution of TFBSs was not biased in the highly conserved $5^{\prime}$ region of intron 1 using a 50bp window (Additional file 2: Figure S2).

\section{CNV of chicken NRG3}

Quantitative PCR (qPCR) using a set of primers (dup_int2F and dup_int2R) specifically amplified the duplicated intron 2 in $\Psi$ NRG3 (Fig. 3a). Relative copy number was largely different among the 3 chicken breeds: RJF showed the lowest copy number, while HJ had a significantly higher number of $\Psi N R G 3$ than the other breeds (Dunn's multiple comparisons test: RJF vs. HJ, $P<0.001$; BB vs. HJ, $P<0.001$; Fig. 3b). Assuming that all RJF chickens had a set of duplicated elements in the genome, the relative copy number of $\Psi N R G 3$ was estimated to be 6.09 in $\mathrm{BB}$ and 34.75 in HJ. BB chicks showed no difference in $\Psi N R G 3$ copy number between 


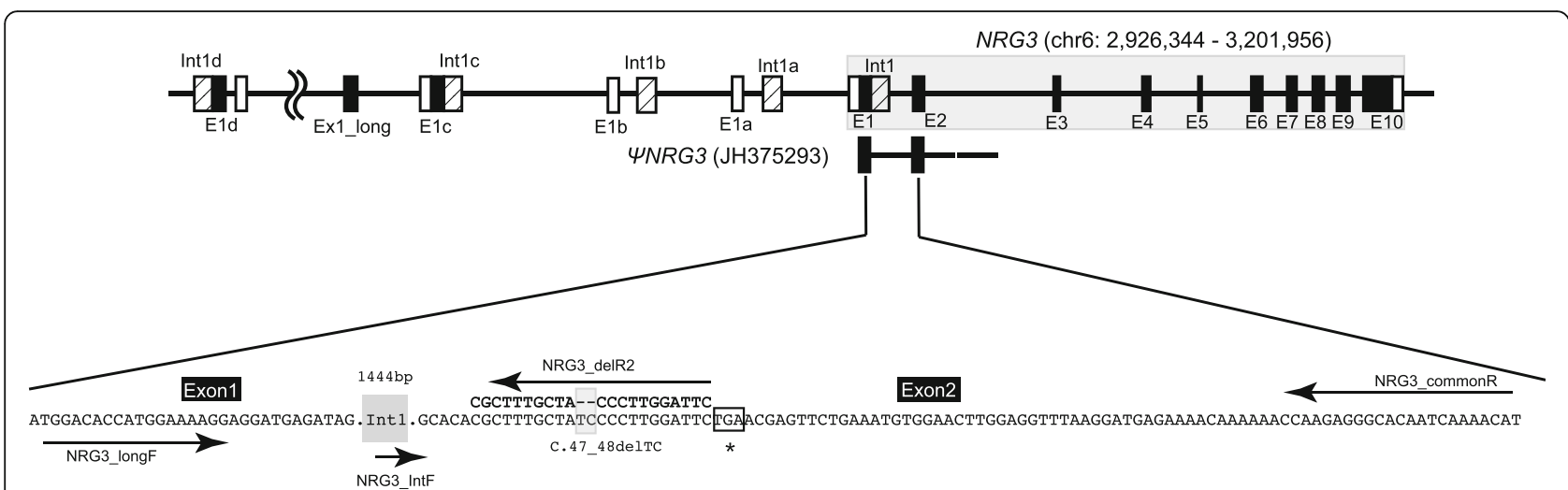

Fig. 1 Schematic gene structure of chicken NRG3. Each coding exon and untranslated region is expressed as a black and open box, respectively. The shaded boxes indicate the $5^{\prime}$ conserved segments of intron 1 (330 bp in length). The size of each box and bar does not correspond to actual sequence length. Four blocks of duplicated exon 1 and flanking segments are shown in the upstream region of the gene (E1a/Int1a, E1b/Int1b, E1c/Int1c, and E1d/Int1d; see Table 1 for more details). Another duplicated segment found in an unknown chromosome of the Red Jungle Fowl genome ( $\Psi$ NRG3; JH375293) is depicted under the schematic gene structure. The enlarged part indicates the positions where oligonucleotide primers were designed for genotyping and sequencing. A putative frameshift deletion (c. 47_48delTC) and premature stop codon are shown in light gray highlight and asterisk, respectively. The dark gray box (Int1) represents the entire intron 1

the $\boldsymbol{d e l}$ and $\boldsymbol{W T}$ groups (Fig. 3c), whereas the copy number of del alleles was significantly higher than that of $W T$ alleles in HJ (Mann-Whitney $U$-test: $U=565.5$, $z=-1.91, P<0.05$; Fig. 3d). The number of $\boldsymbol{d e l}$ alleles was significantly different between $\mathrm{BB}$ and $\mathrm{HJ}$ (MannWhitney $U$-test: $U=97.5, z=3.47, P<0.001$; Table 1; Additional file 3: Table S1).

\section{Structural variation of chicken NRG3 transcripts}

Complementary DNA (cDNA) libraries were constructed by reverse transcription using RNA specimens isolated from 5-day-old HJ chicks. PCR amplification of the cDNA libraries showed different gel electrophoresis patterns.
The intensity of the PCR signal was obviously stronger when a forward primer was chosen in exon 3 than those designed in exons 1 and 2 (Fig. 4a). We isolated cDNA clones and determined the sequences of novel alternatively spliced transcripts in $\boldsymbol{W T}$ and $\boldsymbol{d e l}$ chicks. Variants 1 to 5 were identified from cDNA clones generated by orf_F/orf_R primers, while variants 6 and 7 originated from clones generated by the Ex2_F/orf_R primer set. Exon 2 skipping and intron retention were the major sources of the splice variation in chicken NRG3 (Fig. 4b). Each isoform harboring the retained intron had a PTC that was produced by a shift in the reading frame. Variants 1 and 2 had no PTC in their mRNA sequences and were

Table 1 Highly conserved elements found in the upstream region of chicken NRG3

\begin{tabular}{|c|c|c|c|c|c|c|}
\hline & Region & Start & End & Length (bp) & Strand & Similarity (\%) \\
\hline NRG3 & Exon 1 & $3,201,956$ & $3,201,853$ & 104 & Reverse & \\
\hline$N R G 3$ & Intron $1^{a}$ & $3,201,852$ & $3,201,523$ & 330 & Reverse & \\
\hline E1a & Exon 1 & $3,209,457$ & $3,209,388$ & $70^{\mathrm{b}}$ & Reverse & 94.29 \\
\hline Int1a & Intron $1^{a}$ & $3,207,504$ & $3,207,175$ & 330 & Reverse & 94.85 \\
\hline$E 1 b$ & Exon 1 & $3,217,198$ & $3,217,129$ & $70^{b}$ & Reverse & 97.14 \\
\hline Int1b & Intron $1^{a}$ & $3,215,224$ & $3,214,895$ & 330 & Reverse & 95.15 \\
\hline E1c & Exon 1 & $3,234,535$ & $3,234,437$ & 99 & Reverse & 95.56 \\
\hline Int1c & Intron $1^{a}$ & $3,234,436$ & $3,234,120$ & 317 & Reverse & 92.14 \\
\hline Ex1_long ${ }^{c}$ & Exon 1 & $3,304,770$ & $3,304,436$ & 335 & Reverse & 99.70 \\
\hline E1d_1 & Exon 1 & $5,485,492$ & $5,485,523$ & 32 & Forward & 93.75 \\
\hline E1d_2 & Exon 1 & $5,485,525$ & $5,485,551$ & 27 & Forward & 96.30 \\
\hline E1d_3 & Exon 1 & $5,485,879$ & $5,485,912$ & 34 & Forward & 97.06 \\
\hline Int1d & Intron $1^{a}$ & $5,485,913$ & $5,486,242$ & 330 & Forward & 95.15 \\
\hline
\end{tabular}

${ }^{a}$ The exon 1 adjacent region ( $330 \mathrm{bp}$ ) is considered here, due to its high sequence similarity

${ }^{b}$ Only the 5' UTR is highly conserved

"Truncated sequence of "Exon 1_long" in the CLASS II isoforms (see Fig. 4) 


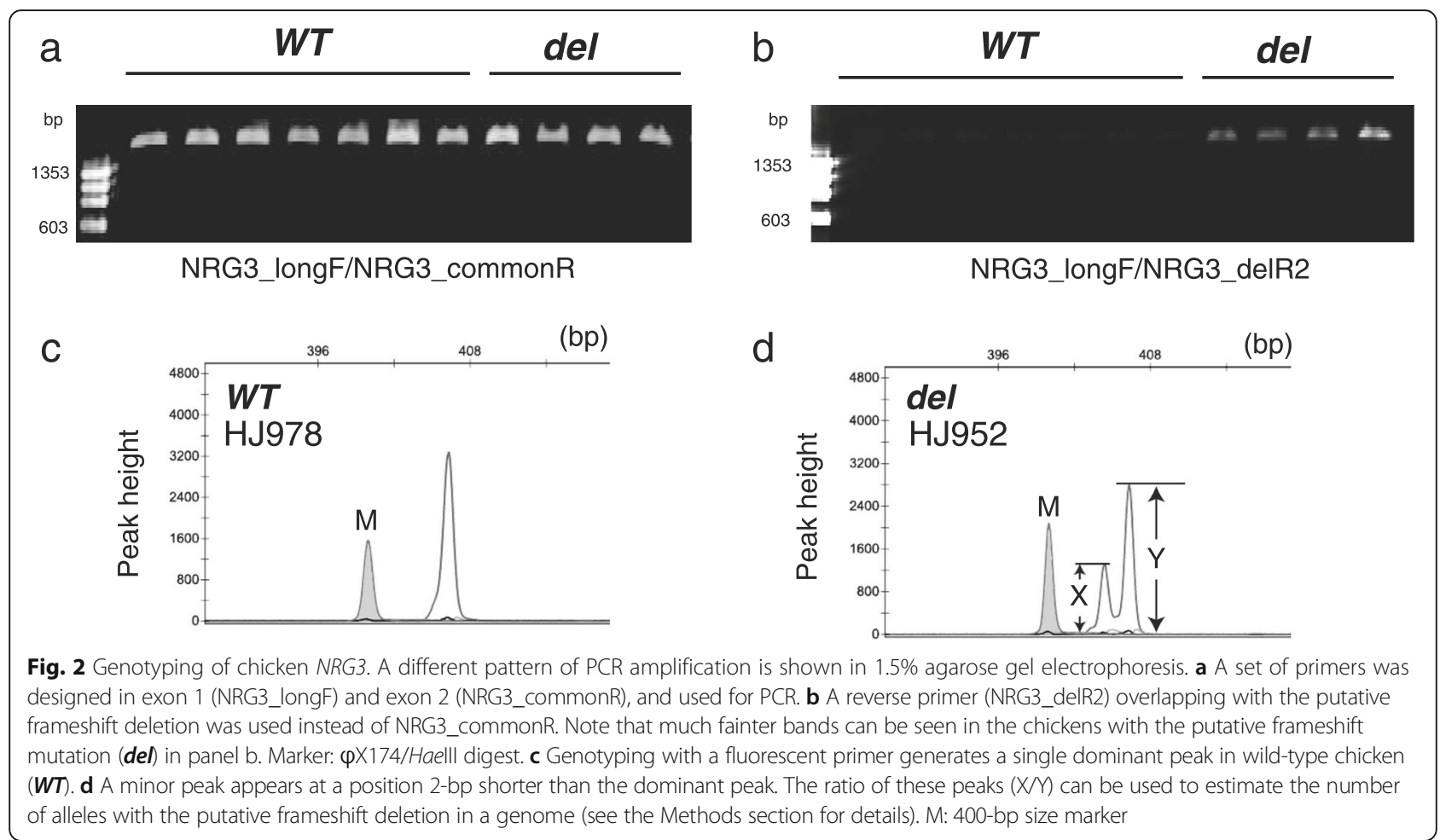

thereby classified as independent isoforms (class III). Nucleotide sequences for NRG3 transcript variants were deposited in the GenBank database under the accession numbers LC175,460 - 1,755,466.

\section{Relative mRNA expression with or without the exon 2 cassette}

Reverse transcription quantitative PCR (RT-qPCR) using exon-specific primers revealed different patterns of relative mRNA expression among the isoform classes. The CLASS I isoform showed no difference in relative expression between the $\boldsymbol{d e l}$ and $\boldsymbol{W T}$ groups (Fig. 5a). The CLASS III isoforms indicated a similar pattern of expression with CLASS I, even though the alternative exon 2 cassette was excluded (Fig. 5b). In the CLASS II isoforms, a significant difference in relative mRNA expression was detected between $\boldsymbol{d e l}$ and $\boldsymbol{W T}$ (Mann-Whitney $U$-test: $U=240$, $z=-2.45, P<0.01$; Fig. $5 \mathrm{c}$ ). There was no correlation of relative gene expression for the CLASS II isoforms with the relative number of $\Psi$ NRG3 between $\mathbf{d e l}$ and WT (del, $R^{2}=0.055 ; W T, R^{2}=0.042 ;$ Fig. 5d).

\section{Discussion}

Chickens are inquisitive birds with intelligence surpassing that of many other domesticated animals. However, they are also sufficiently naive that panic can be induced by environmental noise, especially when they are kept in large numbers. The poultry industry has been searching for effective measures to prevent mass panic in cooperation with molecular scientists. We launched this research project to identify genes or genetic regions that are responsible for panic-like behavior in chicks; chicken NRG3, which has structural mutations in its coding region, is a strong candidate gene for this phenotype.

Yan et al. reported that a frameshift deletion in NRG3 was observed in 5 chicken lines (White Leghorn, White Plymouth Rock, Tibetan, Beijing You, and Rhode Island Red) out of 12 breeds tested [1]. Although they used only 1 individual per breed for sequencing, these

Table $\mathbf{2}$ Chicken NRG3 genotypes and copy number variation of partially duplicated segments

\begin{tabular}{|c|c|c|c|c|c|c|}
\hline \multirow[b]{2}{*}{ Breed } & \multicolumn{4}{|c|}{ Genotype } & \multicolumn{2}{|l|}{ Copy Number } \\
\hline & $n$ & male/female & WT (ratio) & del & Relative $\Psi N R G 3$ & Estimated del \\
\hline$\overline{R J F}$ & 24 & $12 / 12$ & $24(1.00)$ & $0(0)$ & 0.036 & - \\
\hline BB & 44 & $44 / 0$ & $24(0.55)$ & $20(0.45)$ & 0.111 & $4.030\left(2.403^{\mathrm{a}}\right)$ \\
\hline HJ & 60 & $20 / 40$ & $35(0.58)$ & $25(0.42)$ & 1.925 & $61.751\left(5.250^{a}\right)$ \\
\hline
\end{tabular}

$R J F$ red junglefowl, $B B$ Boris Brown, $H J$ Hinai-jidori

${ }^{\text {a }}$ The minimum value is assumed to have a single del allele 


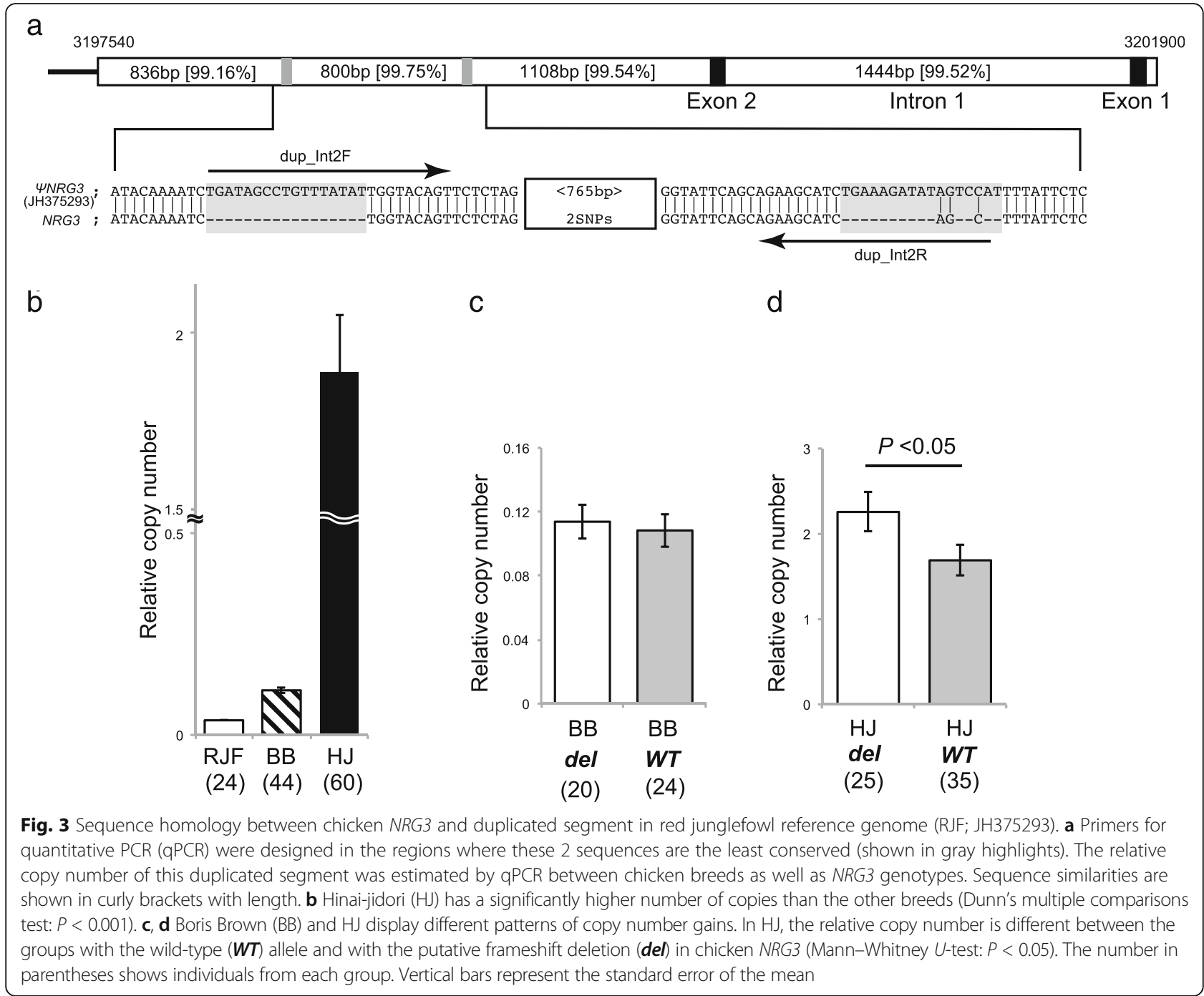

deletion alleles seem to be prevalent mainly in the commercial lines rather than in local breeds. The most plausible scenario for the evolution of chicken NRG3 is that the first duplication of the $5^{\prime}$ part of NRG3 ( NRR3) occurred in the ancestral population of RJF, followed by a rapid increase of its copy number in modern commercial lines. During this process, a frameshift mutation occurred in exon 2 and increased the number of alleles, coinciding with the rapid expansion of $\Psi N R G 3$ in the chicken genome. Considering that Rhode Island Red has had a role in the creation of commercial laying strains, the NRG3 frameshift deletion occurred before or during the line formation process. Intensive selection in a closed colony may help to maintain the frequency of deletion alleles and enable them to become prevalent in chicken breeds. Indeed, our previous study on array comparative hybridization demonstrated that commercial breeds of chicken have a considerable number of breed-specific CNV in their genomes [21]. Although many $\mathrm{CNV}$ loci are thought to be deleterious in the mammalian genome [22], the structural mutations identified in chicken NRG3 seem to be favored by positive selection. To confirm these hypotheses, we need to increase the number of chicken breeds and specimens examined to trace evolutionary changes under selective pressure on the structure of NRG3.

In the present study we identified and characterized 2 structural mutations in chicken NRG3: a putative frameshift deletion in exon $2(\boldsymbol{d e l})$ and CNV in the $5^{\prime}$ part of the gene. These structural mutations are not independent events, because the $\boldsymbol{d e l}$ alleles should be embedded in $\Psi$ NRG3 according to our calculation. This finding is analogous with those obtained by studies of CNV in human neutrophil cytosolic factor 1 (NCF1) and its pseudogenes [23]. Human NCF1 has 2 duplicated copies with a 2-bp deletion in exon 2, which are transcribed differently in various human tissues. It is quite interesting to note that multiallelic $\mathrm{CNV}$ gives rise to most human variation 


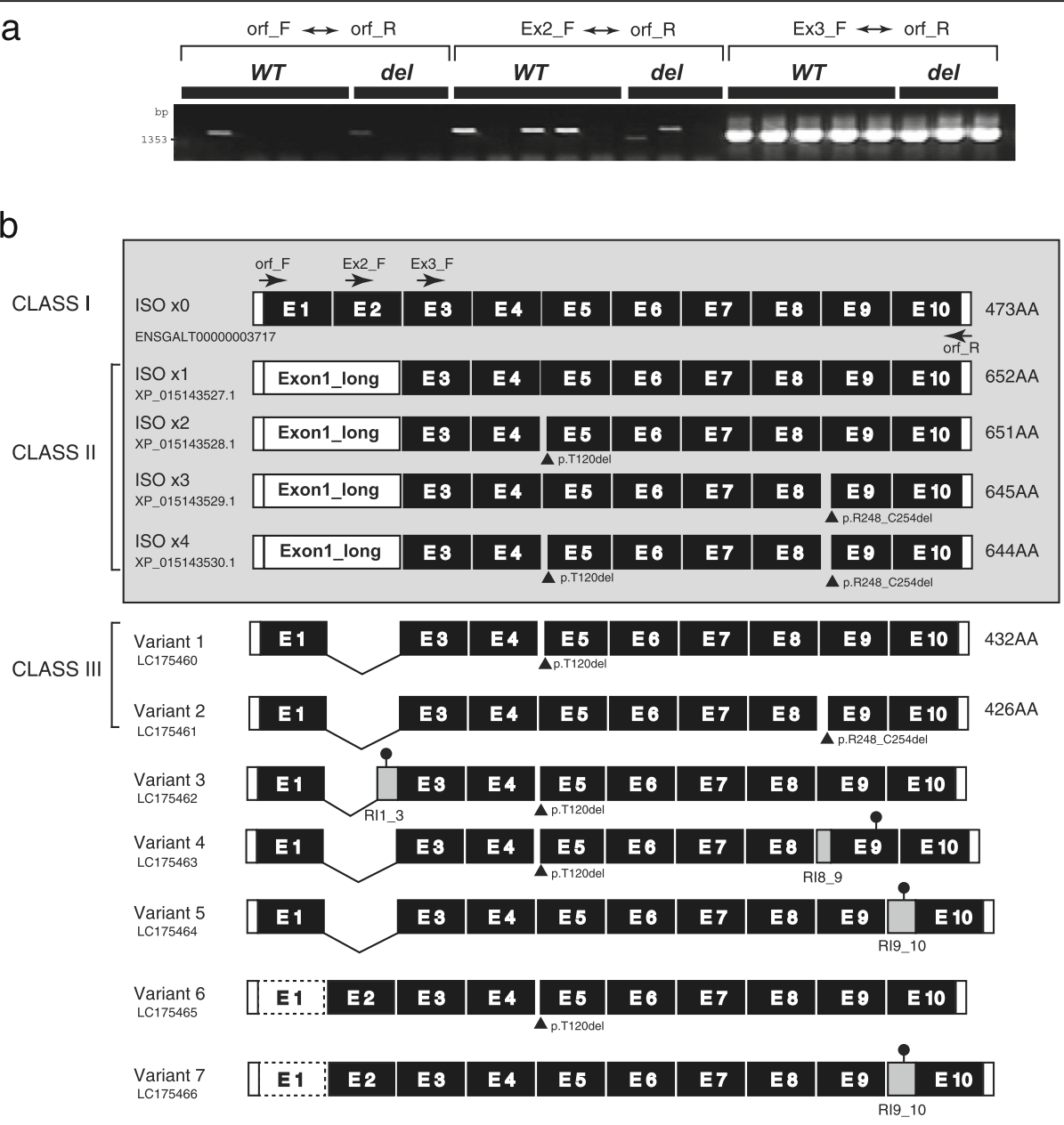

Fig. 4 Schematic representation of transcript variation in chicken NRG3. a cDNA libraries derived from Hinai-jidori were amplified using 3 different sets of primers to estimate the approximate abundance of the $5^{\prime}$ end transcripts by agarose gel electrophoresis. $\mathbf{b}$ NRG3 transcript variants are shown with the reference transcripts (highlighted). The first exon in each ISOX1 to ISOx4 transcript (Exon 1_long) is not annotated as a single independent exon in the galGal5 assembly (NC_006093.4; chr6: 3,826,268 - 3,826,962). The gray blocks depicted in variants 3, 4, 5, and 7 represent the retained introns of various lengths. Note that all transcripts with a retained intron generate a premature termination codon (PTC) in their downstream region. The PTCs and the presumed exon 1 in variants 7/8 are shown as filled circles and a dotted box, respectively

in gene dosage and generates abundant variation in gene expression [24].

Alternative exon skipping events have been observed as potent sources of isoform variation in human NRG3. Several transcripts of chicken NRG3 displayed skipping of exon 2, probably due to a frameshift with a PTC. However, no statistical difference was detected in relative gene expression between the $\boldsymbol{W T}$ and $\mathbf{d e l}$ groups for both CLASS I and III isoforms. If one assumes that the exon 2 skipping event corresponds to a frameshift deletion, CLASS III isoforms should be favored in the del group. A simple explanation for these observations would be the lack of the $\boldsymbol{d e l}$ allele in the true chicken $N R G 3$ gene. In this case, $\boldsymbol{d e l}$ alleles are scattered in only the recently duplicated copies in commercial lines (BB and $\mathrm{HJ}$ ) and would not be involved in NRG3 transcription. This is supported by the fact that exon 2 skipping occurred regardless of the absence of a frameshift deletion in WT chicks (Fig. 5b). Another important finding is that the $\boldsymbol{d e l}$ allele may suppress the expression of the CLASS II isoforms. The observation that the copy number of $\Psi N R G 3$ is significantly larger in chickens with the del allele than in those with the $W T$ allele supports the hypothesis that the biological relevance of the $\boldsymbol{d e l}$ allele is that it acts as a transcriptional regulator under positive selection.

It was somewhat surprising to find a highly conserved sequence block in the $5^{\prime}$ region of intron 1 upstream of chicken NRG3 as well as in the duplicated segments (YNRG3). They show the same or higher levels of sequence conservation compared to the adjacent coding regions. Hence, introns, and especially the first introns, harbor evolutionarily constrained regulatory regions mediating both the level and complexity of gene expression 


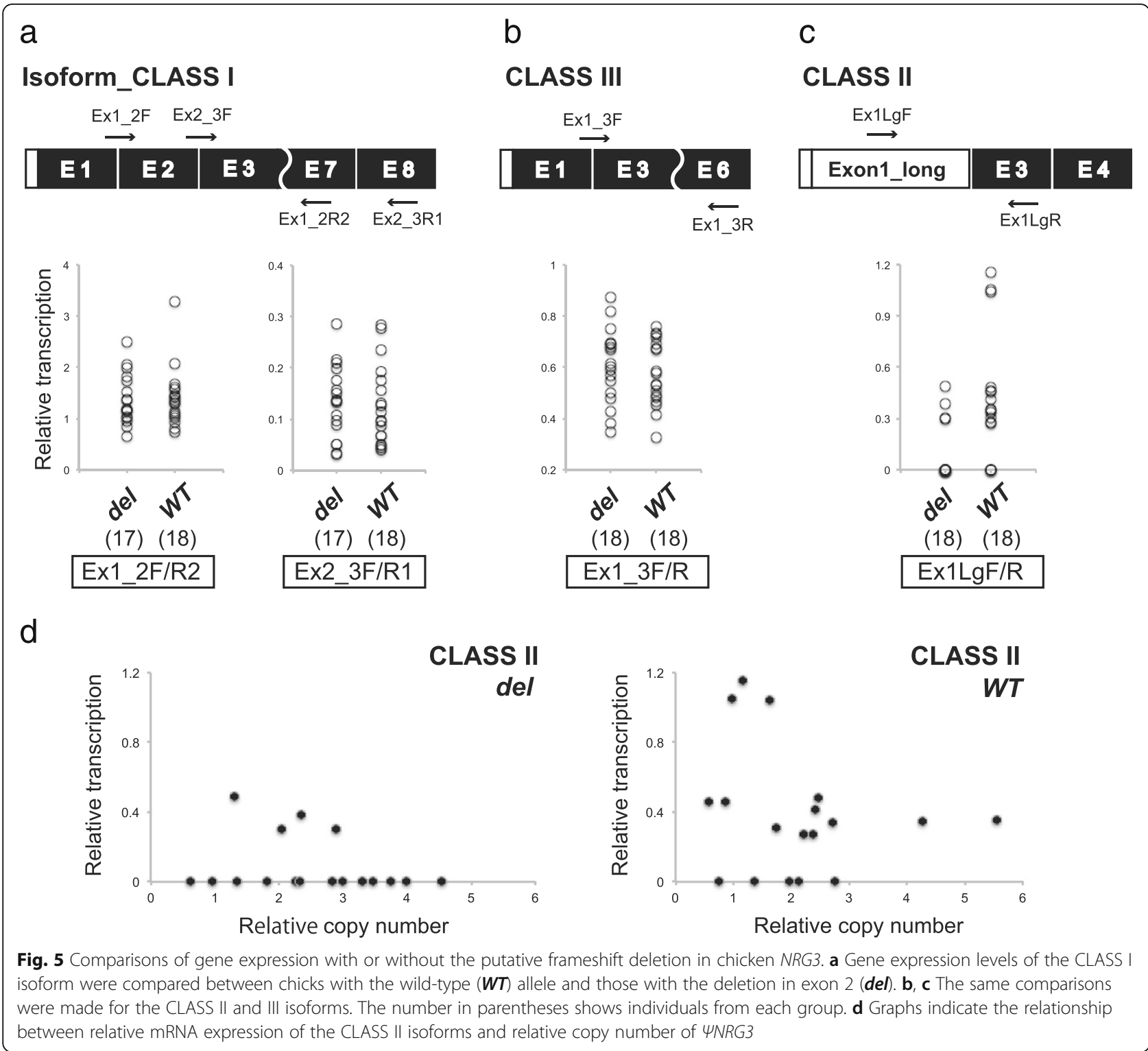

[25]; the $5^{\prime}$ intron of chicken NRG3 may contain transcriptional regulatory elements indispensable for splicing events. As our research did not show a biased distribution of TFBSs in the conserved region of intron 1, other regulatory factors such as histone modification might play an important role in cassette exon inclusion or skipping [26].

There are several studies suggesting that exon 2 of NRG3 might affect the behavioral phenotypes of mice by changing its expression. Loo et al. generated NRG3 mutant mice whose exon 2 was replaced with a neomycin cassette, and demonstrated that an increase in NRG3 expression levels in the medial prefrontal cortex caused an increase in impulsive behavior [27]. Another study also targeting exon 2 of murine NRG3 revealed that mutant mice display decreased freezing behavior and novelty- induced hyperactivity [28]. These findings are important because both hyperactivity and impulsive behaviors are the major tendencies observed in patients with schizophrenia and other mental disorders [29, 30]. Therefore, our findings on the naturally occurring mutations in chicken NRG3 will pave the way for a better understanding of the relationship between NRG3 structural variation coupled with altered expression in the brain and the abnormal behavior of animal models.

\section{Conclusions}

This is the first study specifically examining the gene structure of NRG3 in a non-mammalian vertebrate. The findings of the present study provide important information on the structural mutations that occur naturally in chicken NRG3. However, it is still puzzling how these 
structural components, including a putative frameshift deletion, CNV, alternative exons, and highly conserved introns, interplay with one another to orchestrate the complex expression pattern of NRG3. Regarding NRG3 expression, our study has shown that exon 2 harboring a 2-bp deletion is associated with the downregulation of the expression of CLASS II isoforms. Gene expression profiling data collected from microarray or RNA sequencing will hold the key to elucidating further the genetic factors underlying gene expression heterogeneity in chicken NRG3.

\section{Methods}

\section{Samples and DNA/RNA extraction}

Three breeds of chicken were collected from different research facilities: Nagoya University (RJF; $n=24$ ), Shizuoka University (BB; $n=44)$, and Akita Prefectural Livestock Experiment Station (HJ; $n=60)$. DNA was extracted either from blood $(\mathrm{RJF} / \mathrm{HJ})$ or liver tissue $(\mathrm{BB})$ using commercial DNA extraction kits. Regarding HJ chicks, whole brain was removed from 5-day-old chicks to obtain RNA samples. The animals were decapitated rapidly just before sample collection. Each cerebrum was weighed and sliced into 1-mm-thick coronal sections at $4.0 \mathrm{~mm}$ from the postal edge of the cerebrum. Then, the amygdala regions were punched out with an $18 \times 1 / 2$ gauge blunt needle (NIPRO, Osaka, Japan) using the Atlas of the Chick Brain for reference [31]. Total RNA was extracted using an RNeasy ${ }^{\odot}$ Mini Kit (QIAGEN, Tokyo, Japan). RNA concentration was measured with a Qubit $^{\text {TM }}$ RNA HS Assay Kit (Thermo Fisher Scientific, Tokyo, Japan), and adjusted to $50 \mathrm{ng} / \mu \mathrm{L}$ as templates for RT-PCR.

\section{Genotyping of the NRG3 polymorphism}

We designed primers that flanked the 2-bp deletion to perform conventional PCR (NRG3_longF and NRG3_commonR). Another reverse primer was designed in a position overlapping with the deletion in exon 2 (NRG3_delR2; Fig. 1). Primers were designed using Primer 3Plus online software (http://www.primer3plus.com) for the optimization of primer sequences. The sequences of all oligonucleotide primers used in this study are listed in Additional file 4: Table S2. In each case, PCR was carried out in a $15 \mu \mathrm{L}$ reaction mixture containing G-Taq polymerase (Hokkaido System Science, Sapporo, Japan). The amplification conditions for PCR were: $95^{\circ} \mathrm{C}$ for $2 \mathrm{~min}$, then 30 cycles of $95{ }^{\circ} \mathrm{C}$ for $30 \mathrm{~s}, 60{ }^{\circ} \mathrm{C}$ for $30 \mathrm{~s}$, and $72{ }^{\circ} \mathrm{C}$ for $1 \mathrm{~min}$, with a final extension of $72{ }^{\circ} \mathrm{C}$ for $5 \mathrm{~min}$. For genotyping, a forward primer (NRG3_IntF) was fluorescently labeled with 6carboxyfluorescein (6-FAM), and used for PCR amplification under a slightly modified condition $\left(72{ }^{\circ} \mathrm{C}\right.$ for $30 \mathrm{~s}$ in the extension step). PCR products were analyzed on an ABI 3130xl Genetic Analyzer (Applied Biosystems, Tokyo,
Japan) using the GeneScan ${ }^{\circ}$ 500HD (ROX) size standard (Applied Biosystems).

\section{Search for TFBSs in conserved intron 1}

We used LASAGNA-Search 2.0 [19, 20] to identify TFBSs in intron 1 of chicken NRG3. The JASPER core database was selected for searching matrices (cutoff $p$ value $<0.01)$.

\section{qPCR for copy number estimation}

To estimate the number of duplicated copies of $\Psi N R G 3$ in the chicken genome, we designed a set of primers that bind specifically to the duplicate elements (dup_Int2F and dup_Int2R; see Fig. 2a). qPCR was performed using SYBR Premix ExTaq ${ }^{\mathrm{Tm}}$ II (Takara, Ohtsu, Japan) and Thermal Cycler Dice Real Time System II (Takara). The amplification conditions for qPCR were: $95{ }^{\circ} \mathrm{C}$ for $30 \mathrm{~s}$, then 40 cycles of $95{ }^{\circ} \mathrm{C}$ for $5 \mathrm{~s}, 60{ }^{\circ} \mathrm{C}$ for $30 \mathrm{~s}$, and $72{ }^{\circ} \mathrm{C}$ for $30 \mathrm{~s}$, followed by a melting curve step of $95{ }^{\circ} \mathrm{C}$ for $5 \mathrm{~s}, 60{ }^{\circ} \mathrm{C}$ for $30 \mathrm{~s}$, and $95^{\circ} \mathrm{C}$ for $15 \mathrm{~s}$. After qPCR amplification, each PCR product was electrophoresed in a $2.0 \%$ agarose gel to confirm size and fidelity of product. In all cases, we obtained a single strong band of the expected size (Additional file 5: Figure S3). We used $\beta$ actin $(A C T B)$ as a conventional reference gene for normalization. The relative copy number of $\Psi N R G 3$ was calculated based on the standard curves generated by serial dilution of anonymous DNA as a template.

\section{Calculation of the number of del alleles in each chick}

A combination of relative peak height in fluorescent genotyping and copy number estimated by qPCR was used to calculate the number of $\mathbf{d e l}$ alleles in each individual. Given that all RJF have a set of partially duplicated NRG3 ( NNRG3) in their genome, the number of del alleles can be calculated using the following equation:

Number of $\boldsymbol{d e l}$ alleles $=\left(4 \mathrm{X} \times \mathrm{RCN}_{\text {samples }}\right) /(\mathrm{Y} \times \mathrm{RCNRJF})$,

where $\mathrm{X}$ is the peak height of the 2-bp shorter (del) allele, and $\mathrm{Y}$ is the peak height of the wild-type allele (see Fig. 2d). RCN stands for the relative copy number estimated by qPCR.

\section{Identification of isoform variation}

To isolate transcript variations of chicken NRG3, RT-PCR was performed on each $\mathbf{W T}$ and $\mathbf{d e l}$ sample chosen from the $\mathrm{HJ}$ population. A cDNA library was constructed using $5 \mu \mathrm{g}$ total RNA extracted from the amygdala. Reverse transcription was performed with a random oligo $\mathrm{d}(\mathrm{T})_{18}$ primer and the other reagents supplied in the PrimeScript ${ }^{\mathrm{Tm}}$ RT-PCR Kit (Takara) according to the manufacturer's instructions. Then, first strand CDNA was used for PCR 
amplification with oligonucleotide primers designed in the first and last exons (orfF and orfR; see Fig. 3b). PCR products were electrophoresed on a $1.5 \%$ agarose gel to check size and integrity. Newly synthesized cDNA was diluted with $18 \mu \mathrm{L}$ distilled water and cloned into the $\mathrm{pCR}^{\oplus} 2.1$ $\mathrm{TOPO}^{\circ}$ vector supplied in the $\mathrm{TOPO}^{\circ}$ TA Cloning Kit (Life Technologies, Tokyo, Japan). Escherichia coli DH5a competent cells (Takara) were used for chemical transformation. Insertion was confirmed by PCR amplification with universal vector primers (M13) after plasmid preparation using a QIAprep ${ }^{\oplus}$ Spin Miniprep Kit (QIAGEN).

\section{Validation of relative gene expression}

Two-step RT-qPCR was performed using the same SYBR amplification kits and real-time PCR machine described above. All forward oligonucleotide primers were designed in exon-exon junctions, except for "Exon1_long," as shown in Fig. 4. We used the same set of $A C T B$ primers with the above-mentioned qPCR assay for reference gene amplification. Relative mRNA expression was evaluated by the same manner described above.

\section{Additional files}

Additional file 1 : Figure S1. Alignments of duplicated exons 1 and 2 found in the upstream region of chicken NRG3 and $\Psi$ NRG3. (PDF $400 \mathrm{~kb}$ )

Additional file $\mathbf{2}$ : Figure S2. Distribution of transcription factor binding sites in intron 1 of chicken NRG3. (PDF 568 kb)

Additional file 3 : Table S1. Estimated number of del alleles in each individual of the chicken breeds. (PDF $46 \mathrm{~kb}$ )

Additional file 4 : Table S2. List of the oligonucleotide primers used in this study. (PDF $45 \mathrm{~kb}$ )

Additional file $\mathbf{5}$ : Figure S3. Example electrophoresis gel after $\mathrm{qPCR}$ using dup_Int2F and dup_Int2R. (PDF $382 \mathrm{~kb}$ )

\section{Abbreviations \\ BB: Boris Brown; cDNA: Complementary DNA; CNV: Copy number variation; HJ: Hinai-jidori; Indel: Insertion and deletion; NRG3: Neuregulin 3; PCR: Polymerase chain reaction; PTC: Premature termination codon; qPCR: Quantitative PCR; RJF: Red junglefowl; RT-qPCR: Reverse transcription QPCR; TFBS: Transcription factor binding site; UTR: Untranslated region; WT: Wild-type}

\section{Acknowledgments}

We are grateful to Takao Namikawa for providing the red junglefowl samples. We also thank Hiromi Kobayashi and Yuki Makimura for their assistance with laboratory work and Yuki Sato in the Akita Prefectural Livestock Experiment Station for assisting with tissue collection.

\section{Funding}

This study was financially supported by the FY2015 Kyoto University Research Grand (Start-up) to HA and by the Japan Society for the Promotion of Science Grant-in-Aids for Scientific Research Number 25118005 to MI-M.

\section{Availability of data and materials}

The datasets generated during and/or analyzed during the current study are available from the corresponding author on reasonable request.

\section{Authors' contributions}

Research concept and design: HA. Material collection: DA and HT. Performed the experiments: HA. Data analysis and interpretation: HA. Wrote the paper:
HA. Figure composition: HA. Supervised the research: MI-M. All authors have read and approved of the final manuscript.

\section{Ethics approval}

Sample collection from BB chickens was carried out at Shizuoka University under the approval of the ethics committee (Institutional Animal Care and Use Committee of Shizuoka University approval numbers: 21-5, 22-5, and 23-6). All procedures regarding $\mathrm{HJ}$ chickens were approved by the Kyoto University Animal Experimental Committee (approval number: WRC-2015004A) with permission from the Akita Prefectural Livestock Experiment Station (Animal Experimentation Committee: approval number: 2015-10).

\section{Consent for publication}

Not applicable.

\section{Competing interests}

The authors declare that they have no competing interests.

\section{Publisher's Note}

Springer Nature remains neutral with regard to jurisdictional claims in published maps and institutional affiliations.

\section{Author details}

${ }^{1}$ Wildlife Research Center, Kyoto University, 2-24 Tanaka-Sekiden-cho, Sakyo, Kyoto 606-8203, Japan. ${ }^{2}$ Akita Prefectural Livestock Experiment Station, 13-3 Kaisonumayachi, Jinguji, Daisen, Akita 019-1701, Japan. ${ }^{3}$ Department of Biological Science, Shizuoka University, 836 Ohya, Suruga, Shizuoka 422-8529, Japan. ${ }^{4}$ Wildlife Genome Collaborative Research Group, National Institute for Environmental Studies, 16-2 Onogawa, Tsukuba, Ibaraki 305-8506, Japan.

Received: 10 November 2016 Accepted: 11 July 2017

Published online: 21 July 2017

\section{References}

1. Yan Y, Yi G, Sun C, Qu L, Yang N. Genome-wide characterization of insertion and deletion variation in chicken using next generation sequencing. PLoS One. 2014;9:e104652.

2. Raes J, Van de Peer Y. Functional divergence of proteins through frameshift mutations. Trends Genet. 2005;21:428-31.

3. Zhang D, Sliwkowski MX, Mark M, Frantz G, Akita R, Sun Y, Hillan K, Crowley C, Brush J, Godowski PJ. Neuregulin-3 (NRG3): a novel neural tissue-enriched protein that binds and activates ErbB4. Proc Natl Acad Sci U S A. 1997:94: 9562-7

4. Carteron C, Ferrer-Montiel A, Cabedo H. Characterization of a neural-specific splicing form of the human neuregulin 3 gene involved in oligodendrocyte survival. J Cell Sci. 2006:119:898-909.

5. Balciuniene J, Feng N, lyadurai K, Hirsch B, Charnas L, Bill BR, Easterday MC, Staaf J, Oseth L, Czapansky-Beilman D, et al. Recurrent 10q22-q23 deletions: a genomic disorder on $10 \mathrm{q}$ associated with cognitive and behavioral abnormalities. Am J Hum Genet. 2007:80:938-47.

6. Chen PL, Avramopoulos D, Lasseter VK, McGrath JA, Fallin MD, Liang KY, Nestadt G, Feng N, Steel G, Cutting AS, et al. Fine mapping on chromosome 10q22-q23 implicates Neuregulin 3 in schizophrenia. Am J Hum Genet. 2009:84:21-34

7. Wang YC, Chen JY, Chen ML, Chen $\mathrm{CH}$, Lai IC, Chen TT, Hong CJ, Tsai SJ, Liou YJ. Neuregulin 3 genetic variations and susceptibility to schizophrenia in a Chinese population. Biol Psychiatry. 2008:64:1093-6.

8. Kao WT, Wang Y, Kleinman JE, Lipska BK, Hyde TM, Weinberger DR, Law AJ. Common genetic variation in Neuregulin 3 (NRG3) influences risk for schizophrenia and impacts NRG3 expression in human brain. Proc Natl Acad Sci U S A. 2010;107:15619-24.

9. Morar B, Dragovic M, Waters FA, Chandler D, Kalaydjieva L, Jablensky A. Neuregulin 3 (NRG3) as a susceptibility gene in a schizophrenia subtype with florid delusions and relatively spared cognition. Mol Psychiatry. 2011; 16:860-6.

10. Zeledón M, Eckart N, Taub M, Vernon H, Szymanksi M, Wang R, Chen PL, Nestadt G, McGrath JA, Sawa A, et al. Identification and functional studies of regulatory variants responsible for the association of NRG3 with a delusion phenotype in schizophrenia. Mol Neuropsychiatry. 2015;1:36-46. 
11. Hayes NV, Gullick WJ. The neuregulin family of genes and their multiple splice variants in breast cancer. J Mammary Gland Biol Neoplasia. 2008;13: 205-14.

12. Steinthorsdottir V, Stefansson H, Ghosh S, Birgisdottir B, Bjornsdottir S, Fasquel AC, Olafsson O, Stefansson K, Gulcher JR. Multiple novel transcription initiation sites for NRG1. Gene. 2004;342:97-105.

13. Feuk L, Carson AR, Scherer SW. Structural variation in the human genome. Nat Rev Genet. 2006;7:85-97.

14. Pezer Z, Harr B, Teschke M, Babiker H, Tautz D. Divergence patterns of genic copy number variation in natural populations of the house mouse (Mus musculus domesticus) reveal three conserved genes with major populationspecific expansions. Genome Res. 2015;25:1114-24.

15. Zhou J, Lemos B, Dopman EB, Hartl DL. Copy-number variation: the balance between gene dosage and expression in Drosophila melanogaster. Genome Biol Evol. 2011:3:1014-24

16. Molin AM, Berglund J, Webster MT, Lindblad-Toh K. Genome-wide copy number variant discovery in dogs using the CanineHD genotyping array. BMC Genomics. 2014;15:210.

17. Park C, Makova KD. Coding region structural heterogeneity and turnover of transcription start sites contribute to divergence in expression between duplicate genes. Genome Biol. 2009;10:R10.

18. Kent WJ. BLAT-the BLAST-like alignment tool. Genome Res. 2002;12:656-64.

19. Lee C. LASAGNA: a novel algorithm for transcription factor binding site alignment. BMC Bioinformatics. 2013;14:108

20. Lee C, Huang CH. LASAGNA-search 2.0: integrated transcription factor binding site search and visualization in a browser. Bioinformatics. 2014;30: 1923-5.

21. Abe H, Nagao K, Inoue-Murayama M. Short copy number variations potentially associated with tonic immobility responses in newly hatched chicks. PLoS One. 2013;8:e80205.

22. Berglund J, Nevalainen EM, Molin AM, Perloski M, Consortium L, Andre C, Zody MC, Sharpe T, Hitte C, Lindblad-Toh K, et al. Novel origins of copy number variation in the dog genome. Genome Biol. 2012;13:R73.

23. Brunson T, Wang $\mathrm{Q}$, Chambers I, Song $\mathrm{Q}$. A copy number variation in human NCF1 and its pseudogenes. BMC Genet. 2010:11:13.

24. Handsaker RE, van Doren V, Berman JR, Genovese G, Kashin S, Boettger LM, McCarroll S. Large multiallelic copy number variations in humans. Nat Genet. 2015;47:296-303.

25. Park SG, Hannenhalli S, Choi SS. Conservation in first introns is positively associated with the number of exons within genes and the presence of regulatory epigenetic signals. BMC Genomics. 2014;15:526.

26. Liu H, Jin T, Guan J, Zhou S. Histone modifications involved in cassette exon inclusions: a quantitative and interpretable analysis. BMC Genomics. 2014;15:1148.

27. Loos M, Mueller T, Gouwenberg Y, Wijnands R, van der Loo RJ, Neuro BMPC, Birchmeier C, Smit AB, Spijker S. Neuregulin-3 in the mouse medial prefrontal cortex regulates impulsive action. Biol Psychiatry. 2014;76:648-55.

28. Hayes LN, Shevelkin A, Zeledon M, Steel G, Chen P-L, Obie C, Pulver A, Avramopoulos D, Valle D, Sawa A, et al. Neuregulin 3 knockout mice exhibit behaviors consistent with psychotic disorders. Mol Neuropsychiatry. 2016;2:79-87.

29. Ouzir M. Impulsivity in schizophrenia: a comprehensive update. Aggress Violent Behav. 2013;18:247-54.

30. Itohara S, Kobayashi Y, Nakashiba T. Genetic factors underlying attention and impulsivity: mouse models of attention-deficit/hyperactivity disorder. Curr Opin Behav Sci. 2015;2:46-51.

31. Puelles L, Martinez-de-la-Torre M, Paxinos G, Watson C, Martínez S. The chick brain in stereotaxic coordinates; an atlas featuring neurometric subdivisions and mammalian homologies. New York, USA: Academic Press; 2007.

\section{Submit your next manuscript to BioMed Central and we will help you at every step:}

- We accept pre-submission inquiries

- Our selector tool helps you to find the most relevant journal

- We provide round the clock customer support

- Convenient online submission

- Thorough peer review

- Inclusion in PubMed and all major indexing services

- Maximum visibility for your research

Submit your manuscript at www.biomedcentral.com/submit
Biomed Central 